\title{
Aplicación de Técnicas de Análisis de Conglomerados y Redes Neuronales Artificiales en la Evaluación del Potencial Exportador de una Empresa
}

\author{
Efraín De La Hoz ${ }^{(1) \star}$ y Ludys López Polo(2) \\ (1) Universidad de Cartagena, Facultad de Ciencias Económicas, Programa Administración Industrial. \\ Campus Piedra de Bolívar, Cartagena. Colombia, (e-mail: edelahozg@unicartagena.edu.co). \\ (2) Universidad Simón Bolívar, Facultad de Ingeniería, Programa Ingeniería Industrial. Calle 59 No. 59-81, \\ Barranquilla. Colombia, (e-mail: lulopez@unisimonbolivar.edu.co).
}

Recibido Mar. 15, 2017; Aceptado May. 10, 2017; Versión final Jun. 15, 2017, Publicado Ago. 2017

\begin{abstract}
Resumen
Se presenta una metodología para el análisis de las condiciones competitivas en el comercio exterior de organizaciones empresariales. El análisis abarca las etapas de medición, evaluación y clasificación de las empresas, a partir de la propuesta de 16 factores clave del potencial exportador. También se evalúa la aplicación del análisis de conglomerados para identificar y caracterizar perfiles competitivos y de redes neuronales artificiales para clasificar el potencial exportador. Los resultados muestran la capacidad del análisis de conglomerados y de las redes neuronales artificiales para discriminar niveles competitivos en el potencial exportador. Su aplicación en el sector químico permitió agrupar las empresas en cuatro perfiles competitivos que asocian sus características. Las redes neuronales artificiales mostraron un $85,7 \%$ de capacidad para discriminar y clasificar las empresas según su perfil competitivo.
\end{abstract}

\section{Application of Cluster Analysis Techniques and Artificial Neural Networks for the Evaluation of the Exporting Capability of a Company}

\begin{abstract}
A methodology for the analysis of competitive conditions in foreign trade business organizations is presented. The analysis includes the steps of measuring, evaluation and classification of enterprises considering 16 key factors in export potential. Also, the application of cluster analysis to identify and characterize profiles in the competitive potential is done, and artificial neural networks were employed to classify the export potential. The results show the ability of the cluster analysis and of the artificial neural networks to discriminate competitive levels in export potential. The application of this methodology in the chemical sector, allowed classifying companies in four competitive groups. Also, artificial neural network showed to be capable of classifying and discriminating the competitive profile of a company with a probability of $85.7 \%$.
\end{abstract}

Keywords: export potential; export orientation; competitiveness 


\section{INTRODUCCIÓN}

La globalización de los mercados ha llevado a las organizaciones a esforzarse en comprender los cambios generados por el desarrollo acelerado de nuevas tecnologías, la interacción dinámica de empresas competidoras y el uso del conocimiento para predecir el riesgo y el rendimiento, de manera que se pueda mejorar la toma de decisiones (Bonomie y Añez, 2009). Lo anterior conduce a que las organizaciones deban readaptarse a las nuevas condiciones de los mercados y desarrollar un profundo cambio estructural (Narváez y Fernández, 2008). Así, la interacción y exposición de las empresas al comercio internacional contribuye en gran medida al crecimiento de la productividad del sector, (Olper et al., 2014).

En este sentido, la orientación exportadora es una nueva línea de estudio en la que las organizaciones pueden identificar oportunidades de negocio en el mercado internacional, siendo más competitivas y reduciendo los riesgos asociados a la toma decisiones (Escandón y Hurtado, 2014). Navarro y Acedo (2012) plantean que la vocación hacia la actividad exportadora estará asociada al logro de ventajas competitivas en los mercados, donde la interrelación de variables como la visión, el comportamiento, la percepción y la actitud de directivos en el desarrollo de la dinámica de comercio exterior juega un papel importante. Por su parte, Ahimbisibwe et al., (2013) argumentan la importancia que juega la orientación exportadora en los resultados de las exportaciones, así como la relación existente entre esta orientación y la innovación en el éxito de los negocios, establecen que las empresas mejor preparadas para el manejo de la información relacionada con el comercio exterior, tienen mejor desempeño al traducirse este en mejores oportunidades para satisfacer las expectativas del cliente objetivo.

Diversos autores, han orientado sus investigaciones a identificar factores y condiciones que potencialicen las condiciones competitivas para el comercio exterior. Así, según Escandón y Hurtado (2014), en las Pymes (Pequeñas y mediana empresas) la experiencia laboral de gerentes y propietarios es una variable fundamental en los resultados de procesos de incursión de las empresas en los mercados internacionales, establecen que la experiencia de los empresarios permite madurez a todos los niveles de la organización (estratégicos, tácticos y operativos), lo que incide en mejores condiciones para el éxito, reconocen la cualidad del emprendedor (actitud para asumir riesgos) y la innovación como factores de gran importancia para la competitividad de las empresas y el posicionamiento en mercados internacionales. Cabarcas y Paternina (2011), analizan diversos factores productivos a partir de los cuales establecen diferencias significativas en los perfiles productivos de las empresas exportadoras y no exportadoras. Por su parte, Smith (2005) identifica determinantes ambientales, organizacionales, gerenciales, estratégicos y funcionales como factores relevantes en el desempeño de las empresas exportadoras y (Correia et al., 2009) establecen el efecto de la calidad de la relación en el desempeño de las exportaciones, mostrando que un exportador puede influir positivamente en su propio desempeño adoptando una orientación de relación que a su vez afectará el desempeño de las exportaciones.

Por otra parte, la literatura relacionada con la conceptualización del potencial exportador es escaza, según Obschatko y Blaio (2003) el potencial exportador de una organización, está asociado al crecimiento de las exportaciones producto de su alto valor influenciado por variables internacionales, locales, tecnológicas entre otras. Paredes (2016), la define como el conjunto de condiciones que tiene una empresa para utilizar sus fortalezas, debilidades y aprovechar las oportunidades del mercado internacional en el desarrollo del comercio exterior.

\section{METODOLOGÍA}

En este trabajo, se presenta una metodología para evaluar las empresas de un sector productivo e identificar áreas de oportunidad en el desarrollo el potencial exportador la cual comprende un proceso de medición, evaluación y clasificación de las empresas. Para la investigación se utilizó el análisis de conglomerados, el cual es una técnica de análisis multivariante correspondiente a los métodos de clasificación automática o no supervisada, que busca agrupar los elementos de una muestra en grupos homogéneos, teniendo en cuenta la similitud entre ellos. (Peña, 2002). Para el análisis se utilizó como criterio de eslabonamiento el método de Ward también llamado método de la varianza mínima el cual es un método jerárquico consistente en agrupar de manera ordenada los individuos del más parecido al menos parecido con alta eficacia en estudios de simulación (De la Garza, et al., 2013). El método, mide la suma de cuadrados $W$ de las diferencias de los individuos dentro del cluster (Peña, 2002), esto es:

$$
W=\sum_{g=1}^{G} \sum_{i=1}^{n_{g}}\left(X_{i g}-\overline{X_{g}}\right)\left(X_{i g}-\overline{X_{g}}\right)^{\prime}
$$


Donde $X_{i g}$ es el valor de la variable del individuo $i$ en el grupo $g, \overline{X_{g}}$ es el valor medio del grupo $g, n_{g}$ es el número de individuos del grupo $g, G$ el número total de grupos. Seguidamente, se eligen los grupos que deben unirse mediante:

$\min \frac{n_{a} n_{b}}{n_{a}+n_{b}}\left(\overline{X_{a}}-\overline{X_{b}}\right)^{\prime}\left(\overline{X_{a}}-\overline{X_{b}}\right)$

Siendo $n_{a} y n_{b}$ el número de individuos en el grupo a y b a unirse, $\overline{X_{a}} y \overline{X_{b}}$ el valor medio del grupo a y el grupo b respectivamente. Lo anterior permitió identificar y categorizar perfiles competitivos en el potencial exportador de las empresas en un sector productivo.

Así mismo, se utilizaron las Redes Neuronales Artificiales (RNA), que son modelos matemáticos que replican de manera simplificada el procesamiento de información del cerebro (Martín et al., 2007; Velásquez et al., 2009), para discriminar y clasificar las empresas en un perfil exportador, a partir del cual se identificaron oportunidades de mejora. Para efectos de su aplicación, se utilizó el Perceptrón Multicapa que es un tipo de red neuronal artificial que se caracteriza por su facilidad de implementación. En este, cada neurona de la red está compuesta por un conjunto de entradas $x_{i}$, a las que se les asocia un peso de conexión $w_{i}$, las cuales son procesadas en el cuerpo de la misma mediante una combinación lineal dada por:

$$
y=\sum_{i} w_{i} x_{i}-\theta
$$

Donde, $x_{i}$ representa la variable de entrada asociada al problema y $\theta$ la función entorno o vecindad, el cual se representa comúnmente a través de una función sigmoidea o una función tangente hiperbólica.

Para el desarrollo de la investigación, se tomó como objeto de estudio 65 empresas del Sector Químico del Departamento del Atlántico registradas en Cámara de Comercio de Barranquilla, a las cuales se aplicó el instrumento MFC_PE (Medición de Factores Clave del Potencial Exportador) propuesto por De la Hoz, et al., (2016), el trabajo de campo permitió obtener información de 52 de las empresas de la población objeto de estudio. Las 13 empresas restantes de la población no suministraron información. Las 13 empresas restantes de la población no suministraron información.

EI FC_PE es un instrumento validado para medir el potencial exportador diseñado a partir de la revisión de la literatura relacionada con la orientación exportadora, que integra 16 factores clave en la competitividad exportadora, sistematizados en cinco dimensiones: Financiera (Gestión Financiera (F1), Gestión del Riesgo (F2), Salud Financiera (F3)), Mercado (Conocimiento del Mercado (M1), Comercio exterior (M2), Competitividad del Producto (M3)), Aprendizaje y crecimiento (Gestión de la Información (AC1), Gestión del Conocimiento (AC2), Gestión de Clima Laboral (AC3)), Cliente (Gestión de Proveedores (C1), Gestión de Clientes (C2), Gestión de Requerimientos (C3)) y Procesos internos (Gestión de Productividad (PI1), Gestión de Innovación (PI2), Factores Logísticos (PI3), Gestión de Tecnologías y Operaciones (PI4)).

Seguidamente, con la información de los 16 factores en las empresas del sector químico se aplicó un análisis de conglomerados, para identificar grupos competitivos que integraran niveles competitivos homogéneos que permitieran reconocer características para discriminar su potencial exportador. El mejor agrupamiento se logró utilizando el criterio de eslabonamiento de Ward con medida de distancia euclídea cuadrada. A partir del criterio de distancia entre conglomerados, se definieron 4 grupos con un nivel de distancia 46,592.

Los resultados del análisis de conglomerados se muestran en las Tablas 1 y 2 . En la Tabla 1, se muestra el número de empresas asignadas por conglomerados y la distancia máxima y promedio desde el centroide, y en la Tabla 2 las distancias entre centroides de conglomerados. Comparativamente, las distancias promedio en cada conglomerado son inferiores a las observadas entre conglomerados, verificándose heterogeneidad entre grupos respecto a la homogeneidad dentro de los grupos. La Fig. 1 muestra de manera esquemática el procedimiento, incluyendo sub-pasos y herramientas o técnicas de análisis utilizadas.

A partir de los resultados se categorizaron los perfiles competitivos. El Perfil 1 denominado "Consolidado" clasifica las empresas con las mejores condiciones competitivas y fortalezas para desarrollar el comercio internacional. Para el caso en estudio, corresponde a las 17 empresas asociadas al conglomerado 1, con puntuación promedio de 2,4. En la Tabla 3, se presentan las puntuaciones y rendimientos promedios de este perfil en cada dimensión y en la Fig. 2 los resultados por factor clave. El Perfil 2 "Maduro" clasifica las 16 empresas del conglomerado 3 con la segunda mejor calificación del sector, con puntuación promedio de 1,97. 


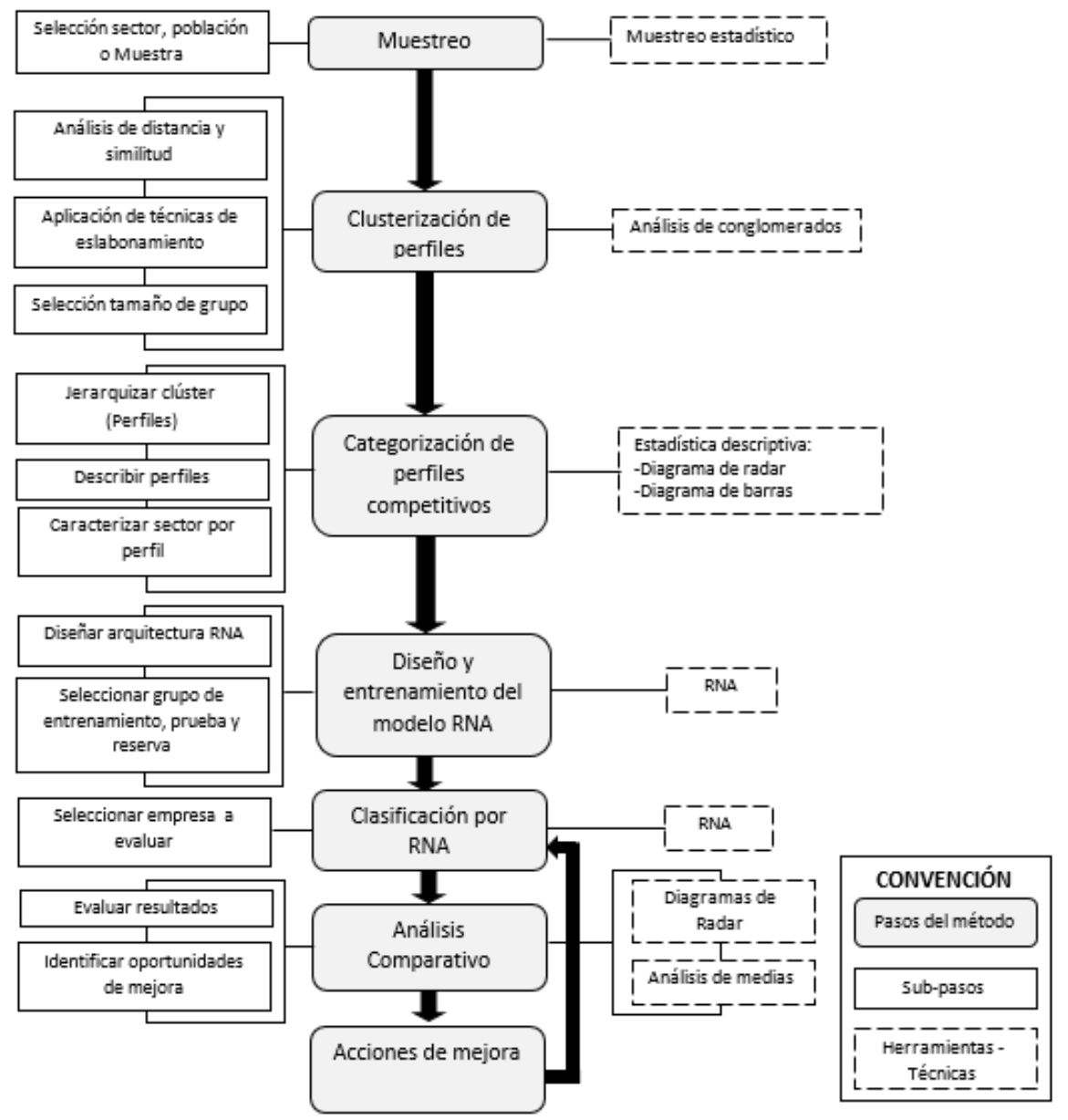

Fig. 1: Flujograma Metodología de Evaluación y Clasificación del Potencial Exportador

Tabla 1: Observaciones por conglomerados

\begin{tabular}{|l|c|c|c|c|}
\hline & $\begin{array}{c}\text { Número de } \\
\text { observaciones }\end{array}$ & $\begin{array}{c}\text { Suma de } \\
\text { cuadrados }\end{array}$ & $\begin{array}{c}\text { Promedio desde } \\
\text { el centroide }\end{array}$ & $\begin{array}{c}\text { Distancia máxima } \\
\text { desde el centroide }\end{array}$ \\
\hline Conglomerado 1 & 17 & 61,1 & 1,79 & 3,56 \\
\hline Conglomerado 2 & 15 & 87,96 & 2,36 & 3,03 \\
\hline Conglomerado 3 & 16 & 72,73 & 2,04 & 3,44 \\
\hline Conglomerado 4 & 4 & 4,51 & 0,99 & 1,59 \\
\hline
\end{tabular}

Tabla 2: Distancias entre centroides de conglomerados

\begin{tabular}{|l|c|c|c|c|}
\hline & Conglomerado 1 & Conglomerado 2 & Conglomerado 3 & Conglomerado 4 \\
\hline Conglomerado 1 & 0 & 3,57 & 2,71 & 6,81 \\
\hline Conglomerado 2 & 3,57 & 0 & 2,43 & 3,67 \\
\hline Conglomerado 3 & 2,71 & 2,43 & 0 & 5,28 \\
\hline Conglomerado 4 & 6,81 & 3,67 & 5,28 & 0 \\
\hline
\end{tabular}

En la Tabla 4, se presentan las puntuaciones y rendimientos promedio del perfil y en la Fig. 3 los resultados por factor clave del perfil 2.

El Perfil 3 "En formación" clasifica las 15 empresas del conglomerado 2, con puntuación promedio de 1,62. En la Tabla 5 se presentan los resultados por dimensión del perfil 3 y en la Fig. 4, las puntuaciones promedio por factores clave del perfil 3. El Perfil 4 "Embrionario" clasifica las 4 empresas del conglomerado 4 con la más baja puntuación $(0,75)$. En este grupo se clasificaron empresas con niveles de rendimiento "bajo" en las cinco dimensiones propuestas, lo que muestra baja condiciones competitivas. En la Tabla 6 se presentan los resultados por dimensión del perfil 4 y en la Fig. 5, las puntuaciones por factores clave que caracterizan las empresas asociadas al perfil 4. 
Tabla 3: Puntuación y rendimiento promedio Perfil 1 "Consolidado

\begin{tabular}{|l|c|c|}
\hline \multicolumn{1}{|c|}{ Dimensión } & $\begin{array}{c}\text { Puntuación } \\
\text { Promedio }\end{array}$ & Rendimiento \\
\hline Financiera & 2,2 & $73 \%$ \\
\hline Procesos Interno & 2 & $67 \%$ \\
\hline $\begin{array}{l}\text { Aprendizaje y } \\
\text { Crecimiento }\end{array}$ & 2,5 & $85 \%$ \\
\hline Clientes & 2,8 & $93 \%$ \\
\hline Mercado & 2,6 & $88 \%$ \\
\hline
\end{tabular}

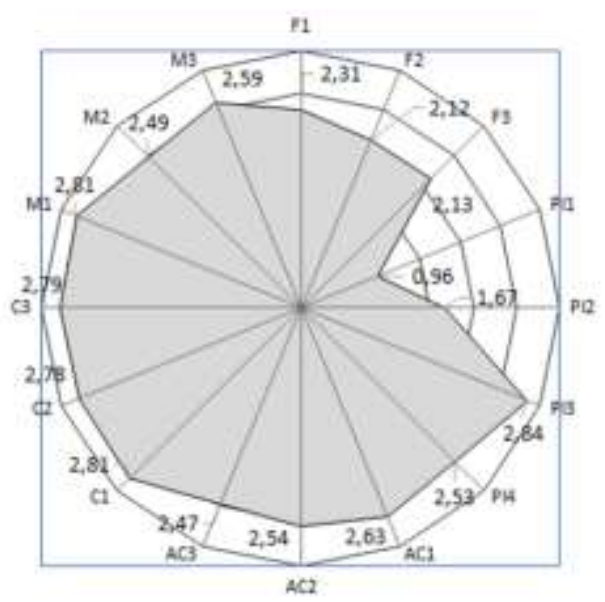

Fig. 2: Perfil 1 "Consolidado

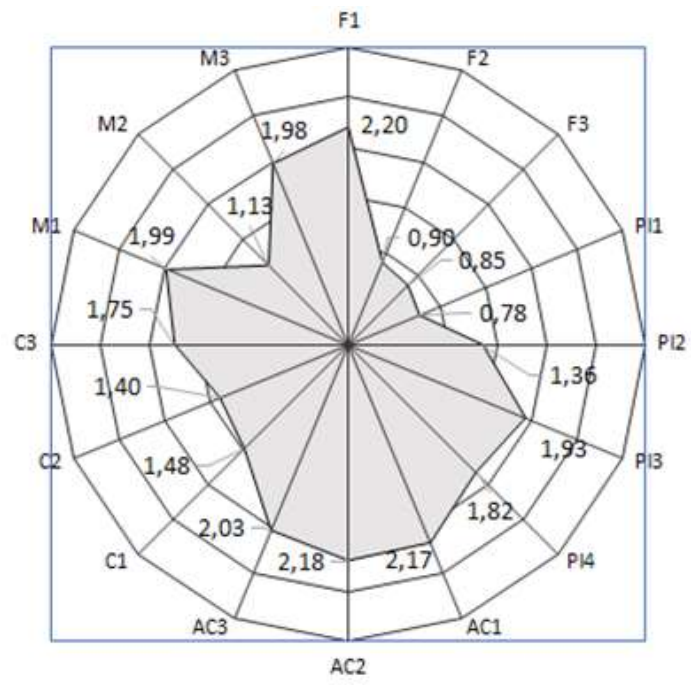

Fig. 4: Perfil 3 "En Formación”

Tabla 5: Puntuación y rendimiento promedio Perfil 3 "En Formación"

\begin{tabular}{|l|c|c|}
\hline \multicolumn{1}{|c|}{ Dimensión } & $\begin{array}{c}\text { Puntuación } \\
\text { Promedio }\end{array}$ & Rendimiento \\
\hline Financiera & 1,3 & $44 \%$ \\
\hline Procesos Interno & 1,5 & $49 \%$ \\
\hline Aprendizaje y Crecimiento & 2,1 & $71 \%$ \\
\hline Clientes & 1,5 & $51 \%$ \\
\hline Mercado & 1,7 & $57 \%$ \\
\hline
\end{tabular}

Tabla 4: Puntuación y rendimiento promedio Perfil 2 Maduro"

\begin{tabular}{|l|c|c|}
\hline \multicolumn{1}{|c|}{ Dimensión } & $\begin{array}{c}\text { Puntuación } \\
\text { Promedio }\end{array}$ & Rendimiento \\
\hline Financiera & 1,1 & $35 \%$ \\
\hline Procesos Interno & 1,8 & $59 \%$ \\
\hline $\begin{array}{l}\text { Aprendizaje y } \\
\text { Crecimiento }\end{array}$ & 2,4 & $80 \%$ \\
\hline Clientes & 2,8 & $93 \%$ \\
\hline Mercado & 1,9 & $63 \%$ \\
\hline
\end{tabular}

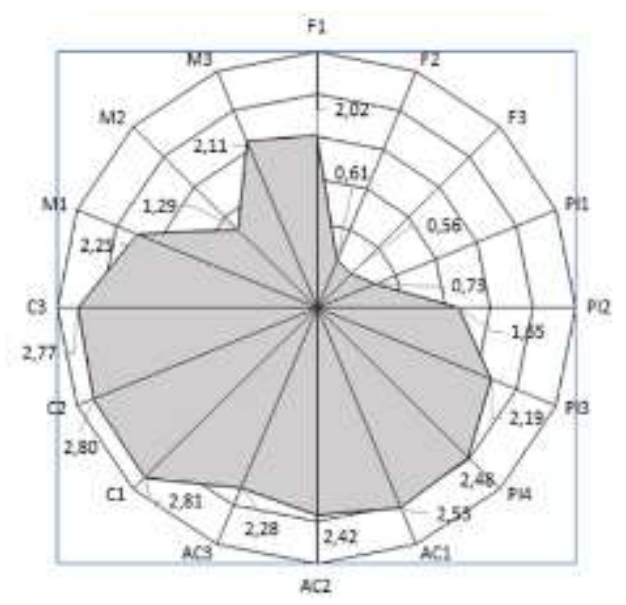

Fig. 3: Perfil 2 "Maduro"

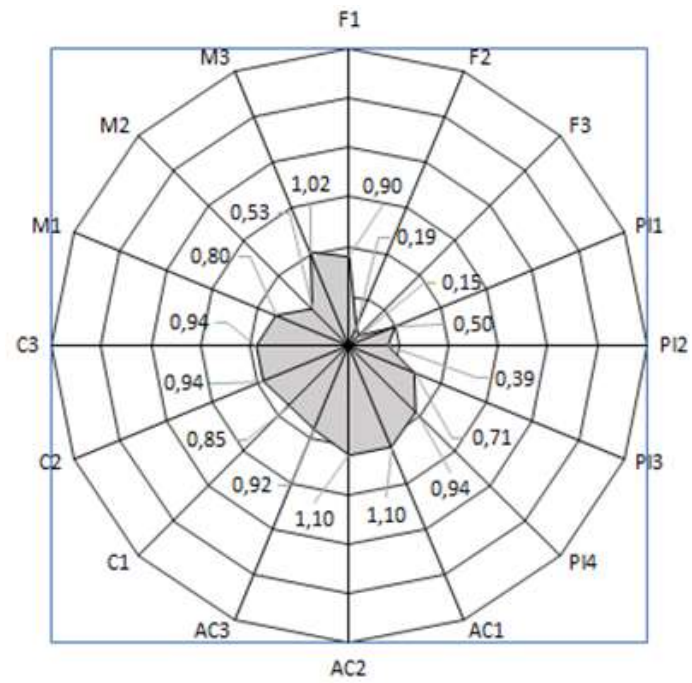

Fig. 5: Perfil 4 "Maduro"

Tabla 6: Puntuación y rendimiento promedio Perfil 4 "Embrionario"

\begin{tabular}{|l|c|c|}
\hline \multicolumn{1}{|c|}{ Dimensión } & $\begin{array}{c}\text { Puntuación } \\
\text { Promedio }\end{array}$ & Rendimiento \\
\hline Financiera & 0,4 & $14 \%$ \\
\hline Procesos Interno & 0,6 & $21 \%$ \\
\hline Aprendizaje y Crecimiento & 1 & $35 \%$ \\
\hline Clientes & 0,9 & $30 \%$ \\
\hline Mercado & 0,8 & $26 \%$ \\
\hline
\end{tabular}


En el cuarto paso, se diseñó el modelo de Red Neuronal Artificial (RNA), se evaluaron arquitecturas del tipo perceptrón multicapa con funciones de activación para las capas ocultas: Tangente hiperbólica o Sigmoide y función de activación para la capa de salida: Identidad, Softmax, Tangente Hiperbólica, Sigmoide a fin de identificar la red que mejor reproduce la clasificación de perfiles. Para la evaluación, se utilizó el software IBM SPSS Statistics 19. En la Tabla 7, se muestran los resultados de clasificación de las arquitecturas evaluadas. Las mejores arquitecturas de RNA presentaron una capacidad del $85,7 \%$ en la muestra de reserva para discriminar y clasificar correctamente una empresa en uno de los perfiles definidos para el potencial exportador.

Tabla 7: Capacidad de clasificación según arquitectura RNA

\begin{tabular}{|c|c|c|c|c|c|c|c|c|c|c|c|c|c|c|c|c|c|}
\hline \multirow{2}{*}{\multicolumn{2}{|c|}{$\begin{array}{c}\text { Número de capas } \\
\text { ocultas } \\
\begin{array}{c}\text { Función de activación } \\
\text { (Capa oculta) }\end{array}\end{array}$}} & \multicolumn{8}{|c|}{1} & \multicolumn{8}{|c|}{2} \\
\hline & & \multicolumn{4}{|c|}{$\begin{array}{l}\text { Tangente } \\
\text { hiperbólica }\end{array}$} & \multicolumn{4}{|c|}{ Sigmoide } & \multicolumn{4}{|c|}{$\begin{array}{l}\text { Tangente } \\
\text { hiperbólica }\end{array}$} & \multicolumn{4}{|c|}{ Sigmoide } \\
\hline \multicolumn{2}{|c|}{$\begin{array}{l}\text { Función de activación } \\
\text { (Capa de salida) }\end{array}$} & 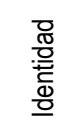 & 希 & 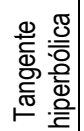 & $\begin{array}{l}\frac{0}{0} \\
\text { हू } \\
\text { के }\end{array}$ & 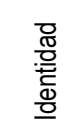 & 希 & 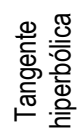 & 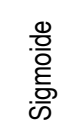 & 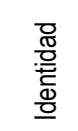 & 离 & 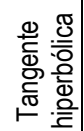 & $\begin{array}{l}\frac{0}{0} \\
\text { है } \\
\frac{0}{\infty}\end{array}$ & 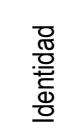 & 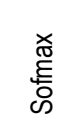 & 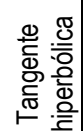 & $\begin{array}{l}\frac{0}{0} \\
\frac{0}{0} \\
\frac{0}{\infty}\end{array}$ \\
\hline $\begin{array}{c}\text { Entrenamiento } \\
(71,2 \%)\end{array}$ & $\begin{array}{c}\% \\
\text { global }\end{array}$ & 100 & 100 & 100 & 100 & 100 & 100 & 100 & 89,2 & 100 & 100 & 100 & 97,3 & 91,9 & 100 & 100 & 100 \\
\hline $\begin{array}{l}\text { Prueba } \\
(15,4 \%)\end{array}$ & $\begin{array}{c}\% \\
\text { global }\end{array}$ & 87,5 & 75 & 50 & 87,5 & 87,5 & 75 & 87,5 & 75 & 75 & 75 & 75 & 87,5 & 75 & 87,5 & 87,5 & 87,5 \\
\hline $\begin{array}{l}\text { Reserva } \\
(13,4 \%)\end{array}$ & $\begin{array}{c}\% \\
\text { global }\end{array}$ & 57,1 & 85 & 71,4 & 85,7 & 71,4 & 85,7 & 42,9 & 85,7 & 57,1 & 42,9 & 57,1 & 57,1 & 71,4 & 85,7 & 85,7 & 85,7 \\
\hline
\end{tabular}

Para valorar el quinto y sexto paso de la metodología, se seleccionó una empresa del sector que en adelante se denomina "muestra", la cual se clasificó en un perfil competitivo mediante RNA. Seguidamente se compararon las métricas (factores) promedios del sector con la empresa. En este análisis comparativo se utilizaron diagramas de radar para visualizar áreas con oportunidades de mejora a partir del perfil de clasificación. El análisis con RNA, permitió asignar la empresa "muestra" en el perfil 1 "Consolidado" con puntuación promedio de 2,05. En la Tabla 8 se sistematizan los resultados en la evaluación de la empresa "muestra" y en la Fig. 6 se contrasta el perfil asignado del sector con la puntuación de la empresa en cada factor clave, con lo cual se identifican oportunidades de mejora en los factores F1, PI1, PI2, AC1, AC2, AC3, M2 y M3 con puntuaciones promedio asociados a los perfiles 3 y 4 .

Tabla 8: Resultados evaluación empresa "muestra"

\begin{tabular}{|c|c|c|c|c|c|c|c|c|c|c|c|c|c|c|c|c|c|}
\hline \multicolumn{3}{|c|}{$\begin{array}{l}\text { Dimensión } \\
\text { Financiera }\end{array}$} & \multicolumn{4}{|c|}{$\begin{array}{c}\text { Dimensión } \\
\text { Procesos Internos }\end{array}$} & \multicolumn{3}{|c|}{$\begin{array}{c}\text { Dimensión } \\
\text { Aprendizaje y } \\
\text { Crecimiento }\end{array}$} & \multicolumn{3}{|c|}{$\begin{array}{c}\text { Dimensión } \\
\text { Clientes }\end{array}$} & \multicolumn{3}{|c|}{$\begin{array}{c}\text { Dimensión } \\
\text { Mercado }\end{array}$} & \multirow{3}{*}{$\begin{array}{c}\frac{0}{0} \\
\frac{1}{2} \\
0 \\
2 \\
2,05\end{array}$} & \multirow{2}{*}{$\begin{array}{l}\frac{8}{8} \\
\frac{\pi}{0} \\
2 \frac{5}{5} \\
1\end{array}$} \\
\hline $\mathrm{F} 1$ & $\mathrm{~F} 2$ & F3 & Pl1 & $\mathrm{Pl} 2$ & $\mathrm{PI} 3$ & $\mathrm{Pl} 4$ & AC1 & AC2 & AC3 & C1 & C2 & C3 & M1 & $\mathrm{M} 2$ & M3 & & \\
\hline 1,5 & 2,5 & 2,25 & 1,67 & 1,33 & 2,75 & 2,67 & 1,75 & 1,75 & 1,5 & 2,25 & 2,25 & 2,25 & 3 & 1,67 & 1,75 & & \\
\hline
\end{tabular}

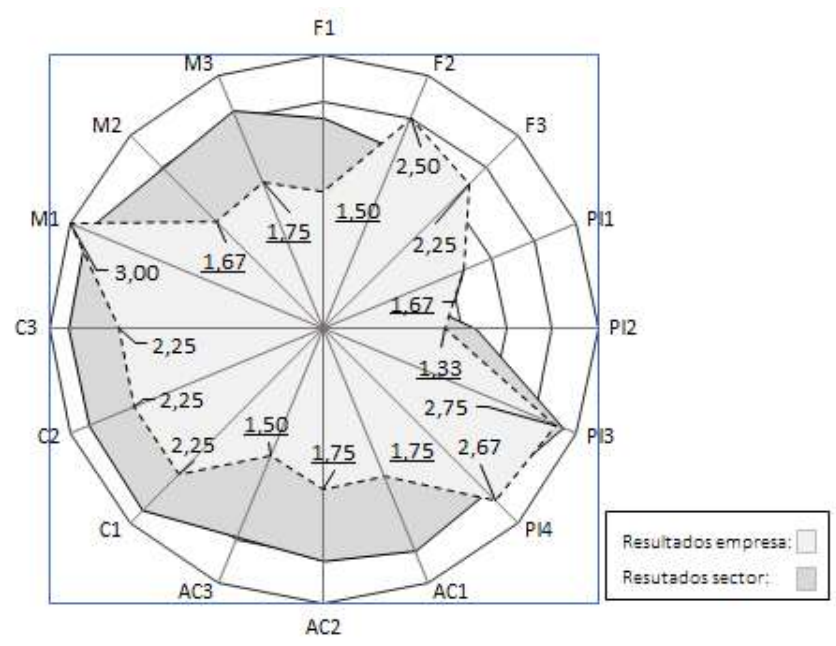

Fig. 6: Empresa "muestra" v-s Perfil Consolidado 


\section{DISCUSION FINAL}

Los procesos de internacionalización de las organizaciones son de vital importancia, dado el contexto de globalización de los mercados y la necesidad de las organizaciones de desarrollar condiciones competitivas para el comercio exterior. La metodología desarrollada en esta investigación, propone el uso del análisis de conglomerados y las redes neuronales artificiales para identificar factores sobre los que se deben concentrar esfuerzos, recursos y controles para mejorar las condiciones competitivas y resultados de la empresa. El análisis de clúster o conglomerados demostró su capacidad para discriminar en el análisis del potencial exportador de las empresas, al establecer niveles competitivos diferenciados a partir de los cuales evaluar las condiciones competitivas exportadora, utilizando factores claves del potencial exportador dados por la Gestión Financiera, Gestión del Riesgo, Salud Financiera, Conocimiento del Mercado, Comercio exterior, Competitividad del Producto, Gestión de la Información, Gestión del Conocimiento, Gestión de Clima Laboral, Gestión de Proveedores, Gestión de Clientes, Gestión de Requerimientos, Gestión de Productividad, Gestión de Tecnologías y Operaciones, Gestión de Innovación y Factores Logísticos, con lo que se definieron métricas para valorar y comparar el potencial exportador a nivel de la empresa y el sector empresarial al que pertenece, lo cual orienta en el desarrollo de condiciones competitivas exportadoras.

Así mismo, se logró establecer la capacidad de las RNA para diferenciar y clasificar las empresas atendiendo perfiles del potencial exportador. Los resultados del análisis, condujeron a identificar 3 modelos de RNA: El primero de 1 capa oculta (función tangente hiperbólica) y 1 capa de salida (función sigmoide), el segundo de 2 capas ocultas y funciones de activación sigmoide en las capa oculta y softmax en la capa de salida y el tercero de 2 capas ocultas con función de activación softmax en la capa oculta y sigmoide en la capa de salida, como las mejores configuraciones de red para discriminar y clasificar el perfil competitivo exportador, con un $100 \%$ de clasificación correcta en la muestra de entrenamiento, $87,5 \%$ en la muestra de prueba y $87,5 \%$ de capacidad en la prueba de reserva, para clasificar correctamente las empresas del Sector Químico del Departamento del Atlántico, lo cual se considera como muy bueno (Fontalvo, et al., 2012).

Lo metodología descrita anteriormente, supone una alternativa relevante en el análisis de sectores productivos, que orienta el diseño de programas y planes por parte de organismos gubernamentales y no gubernamentales para el desarrollo competitivo de estos sectores de la economía. Es importante anotar que no se encontraron referentes de modelos en los que se hiciera este tipo de clasificación y caracterización en el contexto del comercio internacional, a partir de los cuales identificar oportunidades de mejora. El análisis y descripción de los procesos de las organizaciones constituye la base fundamental para iniciar acciones de mejora, dado que se identifican las fallas de los mismos y se pueden dirigir mucho mejor las estrategias (Fontalvo et al., 2010). En este sentido, la caracterización y desarrollo del potencial exportador de las organizaciones permite identificar ventajas comparativas y oportunidades del mercado en el propósito de alcanzar mayores niveles de rentabilidad y competitividad. Lo que muestra la importancia de la metodología propuesta en el propósito de identificar áreas de oportunidad para desarrollar el potencial exportador de las empresas mediante procesos de medición, evaluación y clasificación sustentada en un Perfil de Referencia de Competitividad Exportadora (PRCE), técnicas de análisis multivariante e inteligencia artificial.

\section{CONCLUSIONES}

De acuerdo a los resultados obtenidos, a su análisis y discusión, se pueden obtener las siguientes conclusiones generales:

1.- En el presente trabajo, se presenta una metodología que permite clasificar las organizaciones de un sector empresarial dentro perfiles competitivos para identificar oportunidades mejora y apoyar la toma de decisiones en el direccionamiento y priorización de los recursos.

2.- El análisis de conglomerados demostró capacidad para identificar y caracterizar perfiles competitivos del potencial exportador que constituye una herramienta importante para el análisis de sectores empresariales.

3.- Las Redes Neuronal Artificiales son significativamente sensibles para discriminar niveles competitivos en el potencial exportador con $85,7 \%$ de capacidad para discriminar y clasificar correctamente una empresa en uno de los perfiles definidos en el análisis de conglomerados. Para la capa oculta fueron significativas las funciones hiperbólicas y sigmoide, mientras que en la capa de salida fueron significativas las funciones sofmax y sigmoide.

\section{REFERENCIAS}

Ahimbisibwe, G., Ntayi, J., y Ngoma, M., Export market orientation, innovation and performance of fruit exporting firms in Uganda, European Scientific Journal, 9(4), 295-313 (2013) 
Bonomie, M., y Añez, C., Reflexión teórica de las estrategias flexibilizadoras en el marco de la globalización, Revista Venezolana de Gerencia, 14(48), 592-605 (2009)

Cabarcas, J., y Paternina, C., Aplicación del análisis discriminante para identificar diferencias en el perfil productivo de las empresas exportadoras y no exportadoras del Departamento del Atlántico de Colombia, Revista Ingeniare, 6(10), 33-48 (2011)

Correia, A., Barandas, H., y Plres, P., Applying Artificial Neural Networks to Evaluate Export Performance : A Relational Approach, Review of Onternational Comparative Management, 10(4), 713-734 (2009)

De la Garza, J., Morales, B., y González, B., Análisis Estadístico Mutivariante. Un enfoque teórico y práctico, pp. 150-178, Editorial Mc. Graw Hill, México (2013)

De La Hoz, E., González, Á., y Santana, A., Metodología de Medición del Potencial Exportador de las Organizaciones Empresariales, Información Tecnológica, 27(6), 11-18 (2016)

Escandón, D., y Hurtado, A., Los determinantes de la orientación exportadora y los resultados en las pymes exportadoras en Colombia, Estudios Gerenciales, 30(133), 430-440 (2014)

Fontalvo, T., Vergara, J., y De La Hoz, E., Evaluación del mejoramiento de los indicadores financieros en las empresas del sector almacenamiento y actividades conexas en Colombia por medio de análisis de discriminante, Prospectiva, 10(1), 124-131 (2012)

Fontalvo, T., De La Hoz, E., y Cardona, D., Diseño de un plan de mejoramiento para la cadena de suministro de la empresa Drolitoral S.A. aplicando el Modelo SCOR, Rev. Soluciones de Postgrado EIA, 1(6), 33-53 (2010)

Martín, O., López, M., y Martín, F., Artificial neural networks for quality control by ultrasonic testing in resistance spot welding, Process. Techno., 183, 226-233 (2007)

Narváez, M., y Fernández, G., Estrategias competitivas para fortalecer sectores de actividad empresarial en el mercado global, Revista Venezolana de Gerencia, 13(42), 233-243 (2008)

Navarro, A., y Acedo, F., Efectos de la proactividad exportadora y la orientación al mercado en las operaciones de comercio exterior, Revista Española de Investigación de Marketing, 16(1), 113-133 (2012)

Obschatko, E., y Blaio, M., El perfil exportador del sector agroalimentario argentino. Las profucciones de alto valor. Estudio 1. EG.33.7. Ministerio de Economía de Argentina (2003)

Olper, A., Pacca, L., y Curzi, D., Trade, import competition and productivity growth in the food industry, Food Policy, (49), 71-83 (2014)

Paredes, D., Elaboración del plan de negocios de exportación. Programa de Plan de Negocio, ExportadorPLANEX. Disponible en: https://goo.gl/oTnARL (2016)

Peña, D., Análisis de datos multivariantes, pp. 137-235, Editorial Mc. Graw Hill, Madrid, España (2002)

Smith, D., A Neural Network Classification of Export Success in Japanese Service Firms, Services Marketing Quarterly, 26(4), 95-108 (2005)

Velásquez, J., Franco, C., y García, H., Un modelo no lineal para la predicción de la demanda mensual de electricidad en Colombia, Estudios Gerenciales, 25(112), 37-54 (2009) 